\title{
Identifying and Analyzing Strong Components of an Industrial Network Based on Cycle Degree
}

\author{
Zhiying Zhang, ${ }^{1}$ Xiaozhen Chen, ${ }^{2}$ Wenwen Xiao, ${ }^{1}$ and Guijie $Q{ }^{1}$ \\ ${ }^{1}$ School of Management, Shandong University, Jinan, Shandong 250100, China \\ ${ }^{2}$ Business School, Shandong Normal University, Jinan, Shandong 250014, China \\ Correspondence should be addressed to Xiaozhen Chen; cxz005566@sdnu.edu.cn
}

Received 13 March 2016; Accepted 17 April 2016

Academic Editor: Guo Chen

Copyright (c) 2016 Zhiying Zhang et al. This is an open access article distributed under the Creative Commons Attribution License, which permits unrestricted use, distribution, and reproduction in any medium, provided the original work is properly cited.

\begin{abstract}
In the era of big data and cloud computing, data research focuses not only on describing the individual characteristics but also on depicting the relationships among individuals. Studying dependence and constraint relationships among industries has aroused significant interest in the academic field. From the network perspective, this paper tries to analyze industrial relational structures based on cycle degree. The cycle degree of a vertex, that is, the number of cycles through a vertex in an industrial network, can describe the roles of the vertices of strong components in industrial circulation. In most cases, different vertices in a strong component have different cycle degrees, and the one with a larger cycle degree plays more important roles. However, the concept of cycle degree does not involve the lengths of the cycles, which are also important for circulations. The more indirect the relationship between two industries is, the weaker it is. In order to analyze strong components thoroughly, this paper proposes the concept of circular centrality taking into consideration the influence by two factors: the lengths and the numbers of cycles through a vertex. Exemplification indicates that a profound analysis of strong components in an industrial network can reveal the features of an economy.
\end{abstract}

\section{Introduction}

With the rapid development of the Internet, Internet of Things, and cloud computing technology, data has the potential for an explosive growth. The big-data era, which depends on cloud computing and cloud storage, has arrived. Large scale, diversity, and fast processing speed are the major characteristics of big data. Currently, data research focuses not only on describing individual characteristics but also on depicting the relationships among individuals $[1,2]$. Relational data has been explored in the research of the economic management sector; for example, Acemoglu et al. used a degree sequence of a relational indicator to study the influences of the relationships among different departments on the fluctuation of the total output [3]; Hidalgo et al. studied an industry (product) upgrading issue by studying internet relational stricter among industries (products) [4]; Zhao et al. studied the linkage structure effect of blue economy under the perspective of an industrial network [5]; McNerney et al. studied the structure of interindustry relationships using networks of money flows between industries in 45 national economies [6]. On the basis of an analysis of industrial relational structures using relational data, this paper proposes the concept of circular centrality to detect unknown and potentially useful circular relational data hidden behind Input-Output Tables and study the topological properties of industrial circulation relationships.

In a connected component of an undirected graph, all the vertices are reachable from each other, but this is not necessarily true for a digraph. When there is no path from one vertex to another in a digraph, it is not reachable from the vertex. If there are mutually reachable paths between two vertices, they must be in the same subdigraph, a strong component. A strong component of a digraph, also known as a strongly connected subdigraph, is a maximally induced subdigraph of the digraph. Because any two vertices in a strong component can reach each other, a strong component has features that are different from other components. These make strong components meaningful to a digraph, especially to the one with practical significance such as an industrial network. 


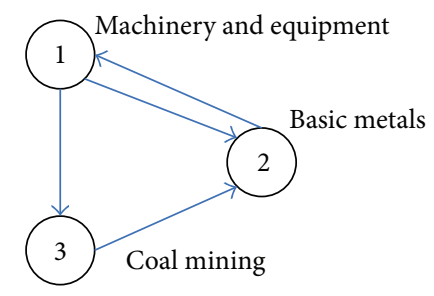

FIGURE 1: Industry circular linkages.

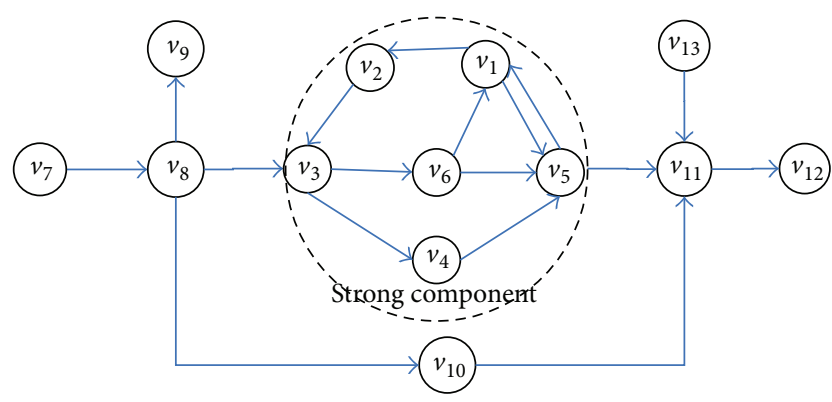

FIgURE 2: An industry network.

With vertices being the sectors and arcs representing significant industrial linkages, an economy can be abstracted into a network-an industrial network. The strong components of an industrial network depict industrial circulation linkages in an economy, where the former sector provides the input for the latter while the output of the latter comes back to the former. As shown in Figure 1, the sectors of coal mining, basic metals, and machinery and the linkages among them make up a strong component, and there are industrial circulation linkages among them. As fundamental linkages, industrial circulation linkages appear in various economic phenomena such as industrial clusters and circular economy [7].

In an industrial network, the general properties of strong components make them an important factor influencing the economy. Since any two sectors in a strong component can reach each other, any change of a sector can be felt by the others, and feedback effects also can influence the initial sector. The effects circulate with diminishing strength, as is shown in Figure 2.

Figure 2 describes an industrial network representing product flows. It contains 13 vertices, and 6 of them are in a strong component, forming vertices set $V_{c}$. If any sector of $V_{c}$ undergoes some changes, such as shrink or expansion, the changes would pass on to all sectors of $V_{c}$ and pass it on to some vertices outside the strong component. For instance, if sector $v_{4}$ alters, all sectors of $V_{c}$ would change accordingly, and the effects circulate again and again over. As for the sectors outside, such as $v_{11}, v_{4}$ would push it along the directed path $v_{4} \rightarrow v_{5} \rightarrow v_{11}$. When the influence circulates within the strong component and reaches $v_{4}$ again, the effects on $v_{11}$ would repeat again with lessened strength.

Any changes of a strong component can influence the circulation of the network. For instance, if $v_{1}$ becomes isolated in Figure 2, there would be no circulation, and any changes of a sector in the network would pass on along unidirectional paths and stop.

Consequently, strong components play significant roles in an economy. Campbell $[8,9]$ recognized the importance and proposed that a strong component could be regarded as a vertex to build the condensation digraph. Some scholars followed this method, such as Morillas et al. [10]. This approach would do a lot to clarify the relationship structure of the whole network but did not involve the internal structure of a strong component. In order to do further researches on strong components, Zhao et al. [11,12] developed the concepts of cycle degree and cycle length distribution of sectors and testified their applicability to show the structure of strong components.

Suppose that $N$ is an industrial network, $V$ is the set of all sectors in $N$, and sector $v \in V$. An industry cycle through $v$ is defined as a directed cycle containing vertex $v$, where no two vertices are the same. It is obvious that an industry cycle, the fundamental circular unit of an industrial network, is a closed path through $v$. Any sector in a strong component is in one or more industry cycles. An industry cycle contains two sectors at least (regardless of loops - arcs beginning and ending at the same vertex) and all sectors of an industrial network at most. In Figure 2, there are four industry cycles through $v_{1}$, that is, $v_{1} \rightarrow v_{5} \rightarrow v_{1}, v_{1} \rightarrow v_{2} \rightarrow v_{3} \rightarrow v_{6} \rightarrow v_{1}, v_{1} \rightarrow v_{2} \rightarrow v_{3} \rightarrow$ $v_{4} \rightarrow v_{5} \rightarrow v_{1}$, and $v_{1} \rightarrow v_{2} \rightarrow v_{3} \rightarrow v_{6} \rightarrow v_{5} \rightarrow v_{1}$.

The cycle degree of a sector in an industrial network, denoted by $d_{c}$, means the number of industry cycles through the sector, describing the number of circular linkages between the sector and the others. As a sector with bigger $d_{c}$ can influence more industry cycles, it has greater driving circulating power. In Figure 2, the cycle degrees of sectors $v_{1}$, $v_{2}, v_{3}, v_{4}, v_{5}$, and $v_{6}$ are $4,3,3,1,3$, and 2, respectively. If $v_{1}$ becomes isolated, there would be no cycles in the network. It can be seen that the sectors with big cycle degrees have more influences on the strong component. Now that the sectors outside the strong component do not exist in any cycles, their cycle degrees are all 0.

Differing from the degree, the cycle degree of a sector depicts the structure information from the whole network. A vertex with small degree is not necessarily with small cycle degree. With in-degree and out-degree both being 1 , maybe a sector is only adjacent to two sectors, but the three sectors are all in multiple cycles, so that the cycle degree of the sector is large still. In Figure 2, with the in-degree and out-degree being $1, d_{c}\left(v_{2}\right)$ is 3 , while $d_{c}\left(v_{4}\right)$ is 1 .

The concept of cycle degree can depict some features of the strong component, but it is not enough. For a vertex, its impact on circulation is related to its cycle degree, but also to the steps in circulations, which is reflected in the lengths of cycles. The effects circulating in a shorter cycle are stronger than in a longer one. If an indicator can capture the two aspects, it is more accurate. For this reason, the concept of circular centrality is proposed in this paper. In addition, in order to better analyze the strong component of an industrial network, the cycle degree of a strong component is presented too.

In order to analyze the effectiveness of these concepts, some practical calculations are made. With more than 30 
years of sustained rapid growth, China's economy has become one of the fastest growing economies in the world. This paper analyzes China's strong components and compares them to those of the US and Japan. The result shows the differences between China and the other two countries.

The organization of this paper is as follows. Section 2 describes the indicators to research strong components based on cycle degree. Section 3 briefly explains the methodology we employed in this study. The next part presents the main empirical results and analyses. Finally, some conclusions are offered.

\section{Relevant Indicators to Analyze Strong Component Based on Cycle Degree}

Strong components of an industrial network can be analyzed from a vertex or from the whole network. From a vertex perspective, as different vertices play different roles in circulations, it is important to analyze these differences. From the whole network, the strong components with different numbers of cycles create different circular effects. In addition, the distribution of sectors with large cycle degree can also show the characteristic of the economy.

2.1. Circular Centrality. The cycle degree of a vertex depicts the number of closed paths through the vertex in circulations. For a vertex, cycle degree can show its effect on circulation, but that is not wholly so. As is seen in Figure 1, there are two cycles through vertex (1), (1) $\rightarrow$ (2) $\rightarrow$ (1) and (1) $\rightarrow$ (3) $\rightarrow$ (2) $\rightarrow$ (1), but they have difference. Vertex (1) gets to itself through two steps along the first cycle, but three steps along the second one. The length of the path affects the linkage between the two sectors. As the path becomes longer, the linkage would be weaker. To a closed directed path, it is obvious that the cycle's length is a factor which can affect the roles of a vertex in circulation. If a vertex is contained in a special long cycle, the effect of the sector on itself would be slow and weak.

In order to express the effect of a sector on circulation, two factors should be taken into consideration, the cycle degree and lengths of cycles through the sector.

Freeman [13] proposed the concept of closeness centrality and suggested that the shorter the distance between a vertex and the others is, the higher closeness centrality it has. A vertex with big cycle degree and short cycles has a high circulation. Considering the two factors, we define the circular centrality of a vertex, which involves the concept of length distribution of cycles [11].

Length distribution of cycles depicts the numbers of cycles with different lengths through a sector. Regardless of loop, the length of the shortest cycle is 2 . As the vertices in a cycle are different, the length of the longest cycle is no more than the number of vertices in the strong component, here assumed to be $m$. Supposing $d_{c}^{k}(v)$ denote the number of $k$ cycles through sector $v$, we get length distribution of cycles $\left(\begin{array}{ccccc}2 & \ldots & k & \ldots & m \\ & \ldots & \ldots & \\ d_{c}^{2}(v) & d_{c}^{k}(v) & d_{c}^{m}(v)\end{array}\right)$. In Figure 2, there are 4 cycles through vertex $v_{1}$, with two cycles of the length of 5 and the others of
2 and 4 . So, the length distribution of industry cycles through $v_{1}$ is $\left(\begin{array}{lll}2 & 4 & 5 \\ 1 & 1 & 2\end{array}\right)$.

Length distribution of cycles involves the lengths of cycles and the corresponding cycle degrees at each length through a vertex. To a certain length, the ratio of cycle degree to its length shows the circular effect. The sum of all ratios depicts the role of the sector in circulation, which is defined as the circular centrality of a sector here. Denote the circular centrality of sector $v$ by $c c_{v}$, and then

$$
c c_{v}=\sum_{k=2}^{m} \frac{d_{c}^{k}(v)}{k} .
$$

The percentage is applicable when the vertices within strong component are compared with each other. The coefficient of circular centrality of sector $v$, denoted by $p_{c c_{v}}$, is the percentage of circular centrality of sector $v$ in those of all sectors in the strong component, and then

$$
p_{c c_{v}}=\frac{c c_{v}}{\sum_{v=1}^{m} c c_{v}} \times 100 \%
$$

The circular centrality of a vertex is a concept based on cycle degree but involves the lengths of cycles. A vertex with big coefficient of circular centrality plays an important role in circulation.

2.2. The Cycle Degree of a Strong Component. Cycles are the fundamental units of a strong component. There must be some cycles in a strong component, at least one. Complete strong components contain the most cycles. The more cycles a strong component contains, the stronger the circularity is. The number of cycles in a strong component is related to the number of vertices and the features of arcs.

The cycle degree of a strong component, denoted by $d_{t}$, is the number of cycles it contains. As the number of vertices in a strong component is constant, it is highly correlated to the arcs there. In general, the cycle degree of a strong component is highly positively correlated to the number of arcs. Sometimes, it is also related to the directions of arcs, and so forth. In Figure 2, if the $\operatorname{arc} v_{1} \rightarrow v_{2}$ becomes $v_{2} \rightarrow v_{1}$, there would remain only one cycle.

As a cycle including several vertices, the cycle degree of the strong component is not the sum of cycle degrees of all vertices there. If there are $k$ vertices in a cycle, the cycle is counted $k$ times, for it is considered in calculating the cycle degree of each vertex in the cycle. In order to calculate it, we can classify all cycles according to their lengths and sum up the cycle degree at each length. Suppose there are $d_{c}^{k}(v) k$ cycles through sector $v$ and $m$ sectors in a strong component, $v=1,2, \ldots, m$. As the number of vertices is that of arcs in a cycle, the number of $k$ cycles in the strong component is $\sum_{v=1}^{m} d_{c}^{k}(v) / k$.

Regardless of loops, arcs beginning and ending at the same vertex, as an industry cycle contains two sectors at least, the length of the shortest cycle is 2 . And that of the longest cycle is $m$, for $m$ sectors in the strong component. So, the cycle 


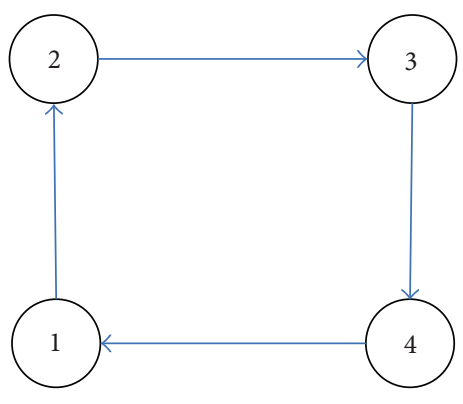

(a) A strong component

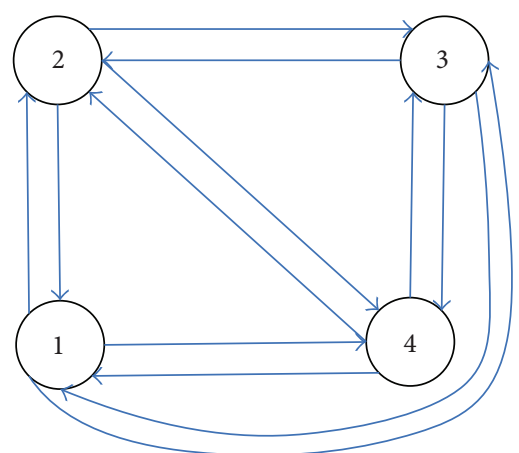

(b) A complete strong component

FIGURE 3: Strong component and the corresponding complete subdigraph.

degree of a strong component, denoted by $d_{t}$, is the sum at each length. That is,

$$
d_{t}=\sum_{k=2}^{m} \frac{\sum_{v=1}^{m} d_{c}^{k}(v)}{k}
$$

The cycle degree of a strong component depicts network connectivity of the strong component. The bigger it is, the stronger the connectivity is.

\section{The Methodology to Identify Strong Components and Calculate the Cycle Degree of a Vertex}

In order to study strong components, we need to identify them from an industrial network first. In addition, calculating the cycle degree of a vertex is the basis to study the strong component. Here, we employ the methodology proposed by Zhao et al. [11] to do these.

3.1. Identifying Strong Components of an Industrial Network. A strong component should be identified from its unique features. All sectors in a strong component can reach one another along directed paths. If arcs are drawn along all directed paths, a strong component would become a complete subdigraph, where there are arcs between any two sectors, as is shown in Figure 3. In the adjacent matrix attached to a complete subdigraph, the entries off the principal dialogue are all "1." According to this, we can distinguish complete subdigraphs from others and identify the strong components accordingly.

In order to identify the strong component of an industrial network, we draw arcs along all directed paths and get a new network, which is called the expansion network here. In the expansion network, the strong components of the primary network become complete strong components. Suppose that the adjacent matrixes of an industrial network and its expansion network are $\mathbf{U}$ and $\mathbf{U}^{*}$, respectively. In the industrial network, if there are $p$ directed walks, length of $k$ from $i$ to $j$, the entries at the intersection of row $i$ and column $j$ of $\mathbf{U}^{k}$ would be $p$ [14]. Suppose that an industrial network contains $n$ vertices; then the length of the longest path (without cycles) of the industrial network will be not more than $n-1$. The sum of the power sequence of the $\mathbf{U}^{k}$ $(k=1, \ldots, n-1)$ matrices gives all walks no more than $k$ steps. When arcs are drawn along all paths, the entries of the corresponding adjacent matrix will become 1 at all positive entries of the sum matrix. So we can get the adjacent matrix $\mathbf{U}^{*}$ from the Boolean summation (i.e., $1+(\#) 1=1$ ) of the power sequence matrices; that is,

$$
\mathbf{U}^{*}=\#\left(\mathbf{U}+\mathbf{U}^{2}+\cdots+\mathbf{U}^{n-1}\right),
$$

where \# denotes Boolean summation.

From $\mathbf{U}^{*}$, we search for all the appropriate $j$ and $k$ for satisfying $u^{*}(k, j)=1$ and $u^{*}(j, k)=1$. All the vertexes $j$ and $k$ are in one complete subdigraph of the industry linkage expansion network; that is, they all belong to one strong component in the industrial network.

3.2. Calculating the Cycle Degree of a Sector. In a strong component, cycle degree of sector $i$ means the number of all cycles through sector $i$, denoted by $d_{c}(i)$. In order to calculate, divide the cycles through sector $i$ into groups according to their length and denote the cycle degree of length $k$ by $d_{c}^{(k)}(i)$. It is obvious that the sum of cycle degrees of all lengths cycles is the cycle degree through sector $i$. Consider

$$
d_{c}(i)=\sum_{k} d_{c}^{(k)}(i)
$$

Ignoring the loops, the shortest cycle is 2 cycles (length of 2 ), and the longest is no more than the number of vertices in the strong component.

From the adjacency matrix of an industrial network, $\mathbf{U}$, we can get the liaisons of any two sectors. If $u_{i j}=1$, there is a direct linkage from sector $i$ to $j$; if $u_{i v_{1}}=u_{v_{1} v_{2}}=\cdots=$ $u_{v_{k-1} j}=1\left(i, v_{1}, v_{2}, \ldots, j\right.$ all differ from one another, so that there is a directed path length of $k$ from sector $i$ to $j$ ), there is an indirect linkage from sector $i$ to $j$, as is shown in Figure 4; otherwise, there is no linkage. When sectors $i$ and $j$ are the same one, the directed path becomes a directed cycle. In other words, there is a $k$ cycle through sector $i$. 

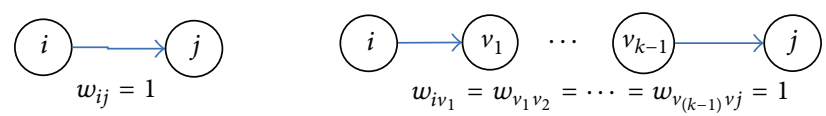

FIgURE 4: Directed path from sector $i$ to sector $j$.

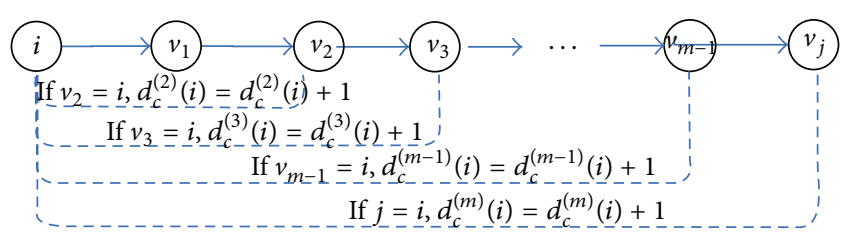

FIgURE 5: Process to calculate cycle degree through sector $i$.

In order to calculate $d_{c}^{(k)}(i)$, we search for the directed closed paths $i \rightarrow v_{1} \rightarrow v_{2} \rightarrow v_{3} \rightarrow \cdots \rightarrow v_{k-2} \rightarrow v_{k-1} \rightarrow i$ $\left(i, v_{1}, v_{2}, \ldots\right.$ all differ from one another) first and then count. The number of all these paths is $d_{c}^{(k)}(i)$, and the sum of all lengths of cycles is $d_{c}(i)$.

As vertices $i, v_{1}, v_{2}, \ldots, v_{k-1}$ are different from each other, the searching process is complex, especially to larger $k$. But an industrial network has its unique characteristics, which make the calculation possible. Firstly, the number of vertices in an industrial network is clear and small, and so is that of a strong component. Secondly, as industrial networks are sparse, if we only calculate unit entries and omit nil entries of the adjacent matrixes, the calculation will be reduced greatly. The algorithm is as follows.

Suppose that there are $m$ sectors in a strong component, and $\mathbf{W}$ is the adjacent matrix attached to the strong component. Let $d_{c}^{(k)}(i)$ be equal to 0 for sector $i$ first. As is shown in Figure 5, the steps are as follows.

Step 1. Search for the suitable $v_{1}$ for $w_{i v_{1}}=1$.

Step 2. Search for the suitable $v_{2}$ for $w_{v_{1} v_{2}}=1$. As no loop is in a strong component, it is certain that $v_{2} \neq v_{1}$. If $v_{2}=i$, there must be a 2 -cycle; then $d_{c}^{(2)}(i) \leftarrow d_{c}^{(2)}(i)+1$; else, go to next step.

Step 3. Search for the suitable $v_{3}$ for $w_{v_{2} v_{3}}=1$, and $v_{3} \neq v_{1}$. If $v_{3}=i, d_{c}^{(3)}(i) \leftarrow d_{c}^{(3)}(i)+1$; else, go to next step.

Step $m-1$. Search for the suitable $v_{m-1}$ for $w_{v_{m-2} v_{m-1}}=1$, and $v_{m-1} \neq v_{1}, v_{2}, \ldots, v_{m-3}$. If $v_{m-1}=i, d_{c}^{(m-1)}(i) \leftarrow d_{c}^{(m-1)}(i)+1$; else, go to next step.

Step $m$. Search for the suitable $v_{m}$ for $w_{v_{m-1} v_{m}}=1$, and $v_{m} \neq$ $v_{1}, v_{2}, \ldots, v_{m-2}$. If $v_{m}=i, d_{c}^{(m)}(i) \leftarrow d_{c}^{(m)}(i)+1$; else, go to next step.

Step $m+1$. Sum up $d_{c}^{k}(i)(k=2, \ldots, m)$; then we get $d_{c}(i)$.

Applying the procedure to all vertices of a strong component, we obtain all cycle degrees.

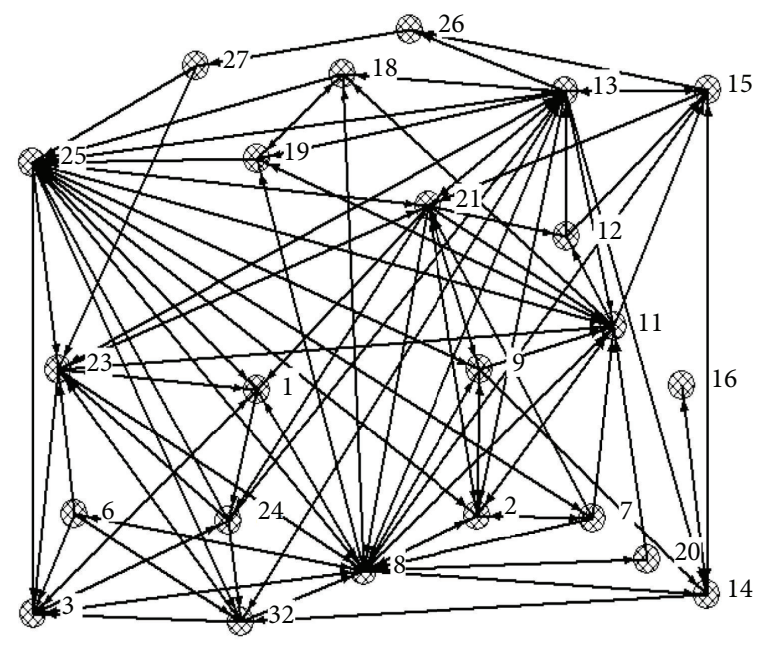

FIgURE 6: Strong component of China's industrial network.

\section{Empirical Calculations and Analyses}

In order to study the effectiveness of the concepts mentioned, some practical calculations are made. China's rapid development has intrigued a great deal of economists to try to understand the process [15-18]. So we try to analyze China's strong components and compare them to those of the US and Japan.

For comparison purposes, the data employed are derived from the OECD Input-Output Database, which is a part of the structural analysis (STAN) database. Data are used from Domestic Input-Output Tables 2005 of China, the US, and Japan (the 2005 Input-Output Tables were the last ones available). The tables all have 37 homogeneous sectors, as presented in Appendix C, making it possible to provide internationally comparable data for research. In order to find the fundamental relationships from the impacts on total production, we calculate the corrected influence coefficients of industrial linkage (Chen and Zhao [19], see Appendix A), on the basis of which industrial networks are constructed (Zhao et al., 2011 [20], see Appendix B).

4.1. Identifying the Strong Component of China's Industrial Network. Based on the methodology of Section 3, we obtain China's strong component. There is only one strong component, containing 23 sectors and 144 arcs, as is shown in Figure 6 . The cycle degree of the strong component of China is 1263356 .

4.2. Cycle Degrees of Sectors in China's Strong Component. The lengths distribution of cycles and cycle degrees of sectors in China are calculated, as is shown in Table 1. It can be seen that, to one sector, when the lengths of cycles are short, as the length is growing, the corresponding cycle degrees rise gradually. When the cycle degree reaches the maximum value, it starts to fall until reaching zero with the increase of the length.

To represent length distribution of industry cycles graphically, with abscissa being the lengths of cycles and ordinate 


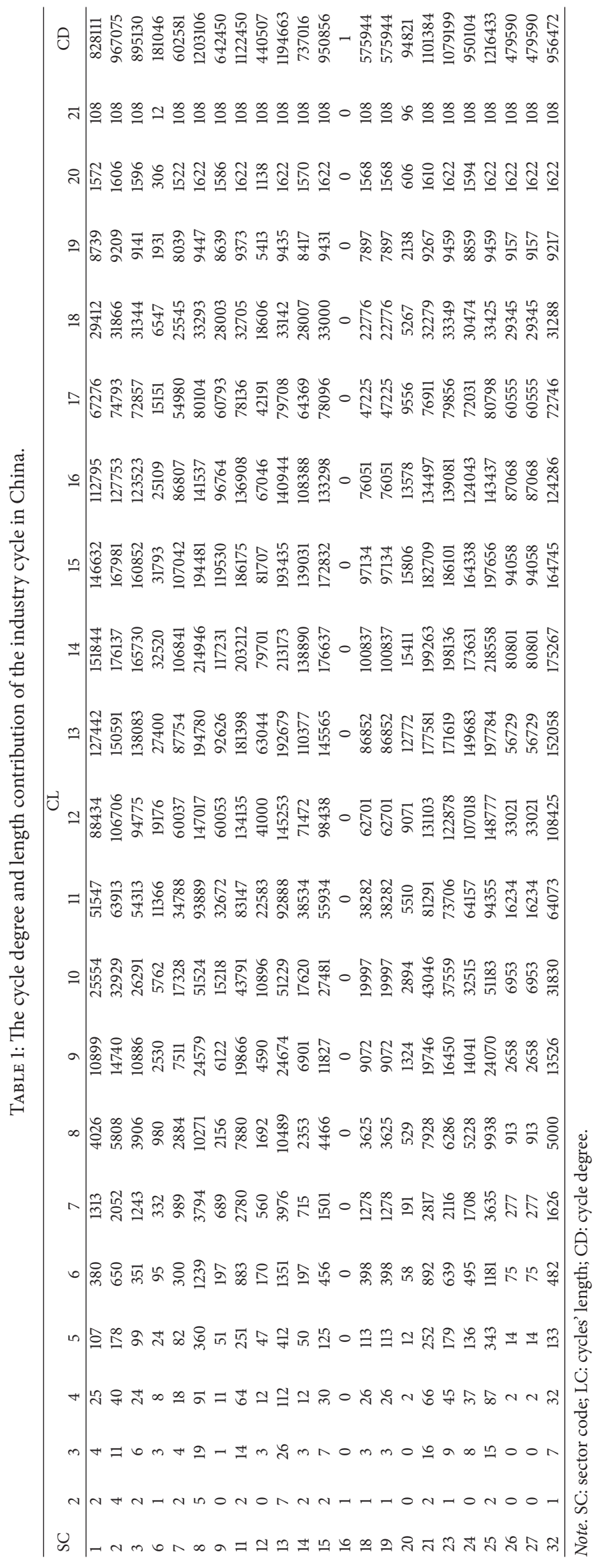




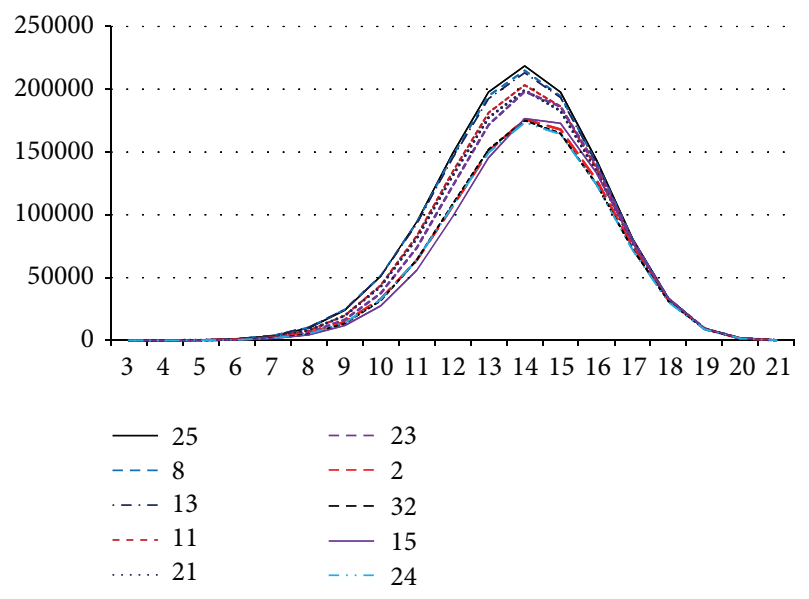

FIGURE 7: Length distribution of cycles of the top ten sectors.

being cycle degrees, we get the line charts of the top ten sectors, as is shown in Figure 7.

From Figure 7, it appears that the curves look like bells, increasing first and then decreasing. In the declining process, several curves overlap gradually. Given closer analysis it can be seen that as cycle becomes longer, there are more sectors in one cycle, so the cycle degrees of the top sectors increase simultaneously.

Dividing curves into halves in the middle parts, it can be seen that the differences of cycle degrees mainly come from the shorter cycles. Since shorter cycles circulate faster than the longer ones, the sectors with larger cycle degrees play more important roles and show greater competitiveness in an economy.

The cycle degree through a sector is an absolute value describing all closed paths through the sector. When we analyze how much a sector impacts the whole structure, relative cycle degree is needed. The relative cycle degree through sector $i$ is the percentage of the cycle degree through sector $i$ in that of the strong component, denoted by $p_{d_{c}(i)}$; then

$$
p_{d_{c}(i)}=\frac{d_{c}(i)}{d_{t}} \times 100 \% \text {. }
$$

The relative cycle degrees of sectors in China are calculated, and the top ten ones are taken out, as is shown in Table 2.

4.3. Strong Component Comparisons among China, the US, and Japan. The cycle degree of the strong component of China is 1263356, while those of the US and Japan are 72712187 and 94706, respectively. It shows that, within an economy, the economic circulation of China is in the middle of the US and Japan. Relatively, Japan is the most dependent on foreign economy, and the US is the weakest.

For comparison, the coefficients of circular centrality and the relative cycle degrees of sectors are calculated and ordered in the three economies. Listing the top ten sectors, we get Table 2.
From Table 2, the top ten cycle degree sectors in the three economies basically share the same rankings with their coefficients of circular centrality. It is thus evident that the sectors with bigger cycle degrees have higher circular centrality generally.

Comparing the ten largest cycle degree sectors in the three economies, we can find that there exist some similarities but more differences in the three economies, as is shown in Figure 8. Overall, there are three common sectors, sectors 23, 25 , and 32 . They all belong to the service sector.

The distributions of the top ten sectors in the three economies are largely in accordance with their characteristics.

In China, the top ten sectors are distributed in three industries, one belonging to the resource industry, five to the manufacturing industry, and four to the service industry. Sector 2 (mining and quarrying) ranks in the top ten only in China. Of the five sectors belonging to the manufacturing industry, four are in the top five. It is evident that the manufacturing industry is the predominant part in China. The US shows its unique characteristics. Of the top ten sectors, nine belong to the service sector. Obviously, the service industry plays a dominant part in the US. Among them, sectors 28 (real estate activities), 31 (research and development), 36 (other community, social, and personal services), and 3 (food products, beverages, and tobacco) are in the top ten sectors only in the US, and sectors 28,31 , and 36 are even in the top five. Japan is somewhere between the US and China. The top ten sectors are both evenly distributed in the manufacturing and the service industry.

\section{Conclusions and Further Research}

Strong components are important components of a digraph for the circulations among vertices. Studying the strong components of an industrial network makes it easier to further identify the structural characteristics of the vertices and the whole network. To describe the strong components of an industrial network more accurately, this paper proposes the concept of circular centrality of a vertex and cycle degree of a strong component on the basis of cycle degree. As circular centrality of a vertex takes into consideration the influence by the lengths and the numbers of cycles through the vertex, it can better describe the real world. Using these indicators, the features of the strong components of China are analyzed and compared with those of the US and Japan.

We can analyze the strong components of an industrial network from two aspects: a vertex and strong component. From the viewpoint of a vertex, circular centrality is a concept involving the cycle degree and lengths of the cycles. Generally, a sector with a higher circular centrality has a stronger circulation and a larger influence on the economy. The study on the strong components of China and the other two economies shows that a vertex with a large cycle degree, with a few exceptions, has a high coefficient of circular centrality. From the viewpoint of a strong component, its cycle degree describes the number of circular paths within it. With a constant vertex number, a strong component with a larger cycle degree circulates stronger. 


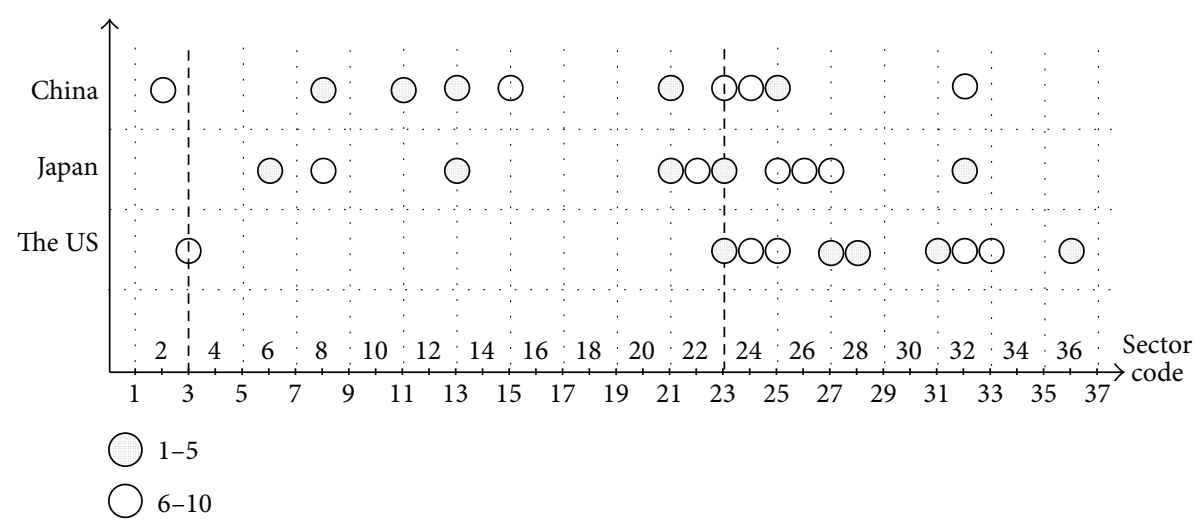

Figure 8: The top ten largest cycle degree sectors of the three economies.

TABLE 2: The top ten sectors of relative cycle degree and coefficients of circular centrality.

\begin{tabular}{|c|c|c|c|c|c|c|c|c|c|c|c|c|c|c|}
\hline \multirow{3}{*}{ SC } & \multicolumn{4}{|c|}{ China } & \multicolumn{5}{|c|}{ Japan } & \multicolumn{5}{|c|}{ The US } \\
\hline & \multicolumn{2}{|c|}{ RCD } & \multicolumn{2}{|c|}{ CCC } & \multirow{2}{*}{ SC } & \multicolumn{2}{|c|}{ RCD } & \multicolumn{2}{|c|}{ CCC } & \multirow{2}{*}{ SC } & \multicolumn{2}{|c|}{$\mathrm{RCD}$} & \multicolumn{2}{|c|}{ CCC } \\
\hline & $(\%)$ & RA & $(\%)$ & RA & & $(\%)$ & RA & $(\%)$ & RA & & $(\%)$ & RA & $(\%)$ & RA \\
\hline 25 & 96.3 & 1 & 7.20 & 1 & 23 & 98.3 & 1 & 8.0 & 1 & 23 & 23 & 1 & 6.24 & 1 \\
\hline 8 & 95.2 & 2 & 7.13 & 2 & 21 & 89.7 & 2 & 7.17 & 2 & 36 & 36 & 2 & 6.23 & 2 \\
\hline 13 & 94.6 & 3 & 7.08 & 3 & 32 & 89.2 & 3 & 7.12 & 3 & 31 & 31 & 3 & 6.17 & 3 \\
\hline 11 & 88.8 & 4 & 6.60 & 4 & 13 & 88.5 & 4 & 7.04 & 4 & 28 & 28 & 4 & 6.01 & 4 \\
\hline 21 & 87.2 & 5 & 6.47 & 5 & 6 & 80.1 & 5 & 6.41 & 5 & 27 & 27 & 5 & 5.85 & 5 \\
\hline 23 & 85.4 & 6 & 6.28 & 6 & 27 & 78.4 & 6 & 6.20 & 6 & 33 & 33 & 6 & 5.79 & 6 \\
\hline 2 & 76.5 & 7 & 5.61 & 7 & 25 & 76.8 & 7 & 6.09 & 7 & 25 & 25 & 7 & 5.1 & 7 \\
\hline 32 & 75.7 & 8 & 5.55 & 8 & 22 & 76 & 8 & 5.99 & 8 & 32 & 32 & 8 & 4.97 & 8 \\
\hline 15 & 75.3 & 9 & 5.45 & 10 & 26 & 70.8 & 9 & 5.52 & 9 & 53 & 53 & 9 & 4.53 & 9 \\
\hline 24 & 75.2 & 10 & 5.51 & 9 & 8 & 67.8 & 10 & 5.3 & 10 & 24 & 24 & 10 & 4.32 & 10 \\
\hline
\end{tabular}

Note. SC: sector code; RCD: relative cycle degree; RA: ranking; CCC: coefficients of circular centrality.

Although the concept of circular centrality of a vertex can describe strong components thoroughly, the calculation methods also have some limitations. Since the calculation process involves circulation, it may take a long time when there are many vertices and arcs in a strong component. As the intermediate inputs of industries are uneven (see Appendix B), the industrial networks are relatively sparse. This makes the method effective in most cases.

Generally, a sector with higher circular centrality has more competitiveness. In order to improve the competitiveness of an economy, we can try to change some linkages through policy guidance to enhance the circular level of the sector and the whole network. It is meaningful to find these linkages and take appropriate action to change them in future research. In addition, there are probably some vertices with more intimate relationships than others within a strong component. Finding these groups will be useful to further analyze the network.

\section{Appendix}

\section{A. The Corrected Influence Coefficients of Industrial Linkage}

From the perspective of the total output of an economy, corrected influence coefficients of industrial linkage delineate the effects of the linkages between two sectors on the total output with the unchanged final demand. The hypothetical extraction method (HEM) is a suitable method for quantitatively calculating the coefficients.

The original idea of the HEM is to extract a sector hypothetically from an economy and examine the impact of the extraction on the economy (Schultz [21]; Cella [22]; Clements [23]; Dietzenbacher and Van Der Linden [24]; Miller and Lahr [25]; Cai and Leung [26]). The differences of total output show effect of the linkages.

Similarly, to assess the linkage effect between two specific sectors, we assume that sector $j$ would purchase import 
goods to substitute for the inputs of sector $i$ completely. Thus, by comparing the total outputs before and after the hypothetical extraction, the linkage between sectors $i$ and $j$ can be calculated. Based on the Leontief model, the industry linkage effect is studied from backward linkages.

Formally, denote the technical coefficient matrix of an economy with $n$ sectors by $\mathbf{A}, \mathbf{A}=\left(a_{i j}\right)_{n \times n}$, and the total output vector by $\mathbf{X}, \mathbf{X}=\left(x_{i}\right)_{n \times 1}$, the final total demand vector by $\mathbf{F}, \mathbf{F}=\left(f_{i}\right)_{n \times 1}$. The impact of a sector on its own is not considered.

In accordance with the Leontief model, the basic balance equation is

$$
\mathbf{X}=\mathbf{A X}+\mathbf{F}
$$

According to HEM, it is assumed that the technical coefficient matrix $\mathbf{A}$ is partitioned into two groups: group one $\left(g_{1}\right)$ being the two sectors to be studied (sectors $i$ and $j$ ) and group two $\left(g_{2}, g_{1}+g_{2}=n\right)$ being the remaining sectors. $\mathbf{X}$ and $\mathbf{F}$ are divided accordingly. Consider

$$
\begin{aligned}
& A=\left[\begin{array}{ll}
A_{11} & A_{12} \\
A_{21} & A_{22}
\end{array}\right], \\
& X=\left[\begin{array}{l}
X_{1} \\
X_{2}
\end{array}\right], \\
& F=\left[\begin{array}{l}
F_{1} \\
F_{2}
\end{array}\right],
\end{aligned}
$$

where

$$
\begin{aligned}
& \mathbf{A}_{\mathbf{1 1}}=\left[\begin{array}{ll}
a_{i i} & a_{i j} \\
a_{j i} & a_{j j}
\end{array}\right]_{2 \times 2} ; \\
& \mathbf{A}_{\mathbf{1 2}}=\left[\begin{array}{lll}
a_{i l} & \cdots & a_{i k} \\
a_{j l} & \cdots & a_{j k}
\end{array}\right]_{2 \times(n-2)} ; \\
& \mathbf{A}_{\mathbf{2 1}}=\left[\begin{array}{ll}
a_{l i} & a_{l j} \\
\cdots & \cdots \\
a_{k i} & a_{k j}
\end{array}\right]_{(n-2) \times 2} ; \\
& \mathbf{A}_{\mathbf{2 2}}=\left[\begin{array}{lll}
a_{l m} & \cdots & a_{l n} \\
\cdots & \cdots & \cdots \\
a_{k m} & \cdots & a_{k n}
\end{array}\right]_{(n-2) \times(n-2)}
\end{aligned}
$$

with $l, k, m, n \neq i, j$.

Equation (A.1) can be rewritten as

$$
\left[\begin{array}{l}
X_{1} \\
X_{2}
\end{array}\right]=\left[\begin{array}{ll}
A_{11} & A_{12} \\
A_{21} & A_{22}
\end{array}\right] \times\left[\begin{array}{l}
X_{1} \\
X_{2}
\end{array}\right]+\left[\begin{array}{l}
F_{1} \\
F_{2}
\end{array}\right]
$$

Solve (A.4):

$$
\begin{aligned}
& \mathbf{X}_{1}=\mathrm{HA}_{12} \mathrm{~B}_{22} \mathrm{~F}_{2}+\mathrm{HF}_{1} \\
& \mathbf{X}_{2}=\mathrm{B}_{22} \mathrm{~F}_{2}+\mathrm{B}_{22} \mathrm{~A}_{21} \mathrm{HA}_{12} \mathrm{~B}_{22} \mathrm{~F}_{2}+\mathrm{B}_{22} \mathrm{~A}_{21} \mathrm{HF}_{1},
\end{aligned}
$$

where $\mathbf{B}_{\mathbf{2 2}}=\left[\mathbf{I}-\mathbf{A}_{\mathbf{2 2}}\right]^{-1}, \mathbf{H}=\left[\mathbf{I}-\mathbf{A}_{\mathbf{1 1}}-\mathbf{A}_{\mathbf{1 2}} \mathbf{B}_{\mathbf{2 2}} \mathbf{A}_{\mathbf{2 1}}\right]^{-1}$, and $\mathbf{I}$ denotes the identity matrix.

Now, suppose that the input from sector $i$ to sector $j$ is extracted from the economy; that is, $a_{i j}=0 . \mathbf{A}_{11}$ becomes $\mathbf{A}_{11}^{\prime}, \mathbf{A}_{11}^{\prime}=\left[\begin{array}{cc}a_{i i} & 0 \\ a_{j i} & a_{j j}\end{array}\right]_{2 \times 2}$, and $\mathbf{X}$ becomes $\mathbf{X}^{\prime}$. Equation (A.4) can be expressed as

$$
\left[\begin{array}{l}
X_{1}^{\prime} \\
X_{2}^{\prime}
\end{array}\right]=\left[\begin{array}{ll}
A_{11}^{\prime} & A_{12} \\
A_{21} & A_{22}
\end{array}\right] \times\left[\begin{array}{l}
X_{1}^{\prime} \\
X_{2}^{\prime}
\end{array}\right]+\left[\begin{array}{l}
F_{1} \\
F_{2}
\end{array}\right] .
$$

Solve (A.6):

$$
\begin{aligned}
& \mathbf{X}_{1}^{\prime}=\mathbf{H}^{\prime} \mathbf{A}_{12} \mathbf{B}_{22} \mathbf{F}_{2}+\mathbf{H}^{\prime} \mathbf{F}_{1} \\
& \mathbf{X}_{2}^{\prime}=\mathbf{B}_{22} \mathbf{F}_{2}+\mathbf{B}_{22} \mathbf{A}_{21} \mathbf{H}^{\prime} \mathbf{A}_{12} \mathbf{B}_{22} \mathbf{F}_{2}+\mathbf{B}_{22} \mathbf{A}_{21} \mathbf{H}^{\prime} \mathbf{F}_{1}
\end{aligned}
$$

where $\mathbf{H}^{\prime}=\left[\mathbf{I}-\mathbf{A}_{11}^{\prime}-\mathbf{A}_{\mathbf{1 2}} \mathbf{B}_{\mathbf{2 2}} \mathbf{A}_{\mathbf{2 1}}\right]^{-1}$.

The difference between (A.5) and (A.7), denoted by ILE $\mathrm{I}_{i j}$, means the industry linkage effect of sectors $i$ and $j$. Consider

$$
\begin{aligned}
& \mathrm{ILE}_{i j}=\mathbf{k}\left(\mathbf{X}-\mathbf{X}^{\prime}\right)=\left[\mathbf{k}_{\mathbf{1}}\left(\mathbf{H}-\mathbf{H}^{\prime}\right)\right. \\
& \left.+\mathbf{k}_{\mathbf{2}} \mathbf{B}_{\mathbf{2 2}} \mathbf{A}_{\mathbf{2 1}}\left(\mathbf{H}-\mathbf{H}^{\prime}\right)\right] \mathbf{F}_{\mathbf{1}}+\left[\mathbf{k}_{\mathbf{1}}\left(\mathbf{H}-\mathbf{H}^{\prime}\right) \mathbf{A}_{\mathbf{1 2}} \mathbf{B}_{\mathbf{2 2}}\right. \\
& \left.\quad+\mathbf{k}_{\mathbf{2}} \mathbf{B}_{\mathbf{2 2}} \mathbf{A}_{\mathbf{2 1}}\left(\mathbf{H}-\mathbf{H}^{\prime}\right) \mathbf{A}_{\mathbf{1 2}} \mathbf{B}_{\mathbf{2 2}}\right] \mathbf{F}_{2},
\end{aligned}
$$

where $\mathbf{k}, \mathbf{k}_{\mathbf{1}}$, and $\mathbf{k}_{\mathbf{2}}$ are row summation vectors for $\mathbf{g}, \mathbf{g}_{1}$, and $\mathbf{g}_{2}$.

The corrected influence coefficient between sectors $i$ and $j$, ilcic $_{i j}$, is the percentage of $\operatorname{ILE}_{i j}$ in the total output. Consider

$$
i l c i c_{i j}=\frac{\mathrm{ILE}_{i j}}{\mathbf{k X}} \times 100 \% \text {. }
$$

\section{B. Constructing the Industrial Network}

The industrial network is a fundamental model to analyze economic structures. With information in Input-Output Tables, the industrial network can be constructed with sectors being vertices, while arcs (directed edges) represent industrial linkages. The numeric values matrices, such as inputoutput quantitative transactions, or coefficients calculated from them, such as corrected influence coefficients, depict various relationships and determine the existence of arcs between sectors. In principle, with a defined threshold value, a numeric values matrix can be transferred into an adjacency matrix attached to a directed graph, with all positive entries larger than or equal to the filter to unity and the rest of the entries to zero.

It can be observed that the intermediate inputs of any sector are uneven. In the technical coefficient matrix $\mathbf{A}$ of any economy, only few $a_{i j}$ values are large. For instance, in Chinese IO tables for the year $2005(37 \times 37)$, the 53 highest intermediate flows comprise $50 \%$ of the total intermediate consumption, while the remaining ones (1316) account for the other $50 \%$. Nonuniform inputs make the industrial networks determined by several important relations.

Considering that the inputs have obvious inflection point, Zhao et al. [20] proposed the method to establish networks with Weaver-Thomas index. 
TABLE 3: OECD sector classification.

\begin{tabular}{|c|c|}
\hline Code & Sector \\
\hline 1 & Agriculture, hunting, forestry, and fishing \\
\hline 2 & Mining and quarrying \\
\hline 3 & Food products, beverages, and tobacco \\
\hline 4 & Textiles, textile products, leather, and footwear \\
\hline 5 & Wood and products of wood and cork \\
\hline 6 & Pulp, paper, and paper \\
\hline 7 & Coke, refined petroleum products, and nuclear fuel \\
\hline 8 & Chemicals and chemical products \\
\hline 9 & Rubber and plastics products \\
\hline 10 & Other nonmetallic mineral \\
\hline 11 & Basic metals \\
\hline 12 & $\begin{array}{l}\text { Fabricated metal products except machinery and } \\
\text { equipment }\end{array}$ \\
\hline 13 & Machinery and equipment n.e.c \\
\hline 14 & Office, accounting, and computing machinery \\
\hline 15 & Electrical machinery and apparatus n.e.c \\
\hline 16 & Radio, television, and communication equipment \\
\hline 17 & Medical, precision, and optical instruments \\
\hline 18 & Motor vehicles, trailers, and semitrailers \\
\hline 19 & Other transport equipment \\
\hline 20 & Manufacturing n.e.c; recycling \\
\hline 21 & Electricity, gas, and water supply \\
\hline 22 & Construction \\
\hline 23 & Wholesale and retail trade; repairs \\
\hline 24 & Hotels and restaurants \\
\hline 25 & Transport and storage \\
\hline 26 & Post and telecommunications \\
\hline 27 & Finance and insurance \\
\hline 28 & Real estate activities \\
\hline 29 & Renting of machinery and equipment \\
\hline 30 & Computer and related activities \\
\hline 31 & Research and development \\
\hline 32 & Other business activities \\
\hline 33 & Public admin and defense; compulsory social security \\
\hline 34 & Education \\
\hline 35 & Health and social work \\
\hline 36 & Other community, social, and personal services \\
\hline 37 & Private households with employed persons \\
\hline
\end{tabular}

Source: This sector classification uses the latest version of the OECD IO tables: 2005 Edition. http://stats.oecd.org/ to obtain the 2005 edition of OECD input-output tables for free.

The Weaver-Thomas index is an effective tool for finding the significant index developed by Weaver first and improved by $\mathrm{O}$. Thomas later. Comparing an observed distribution with an assumed one, the closest approximation distribution is established to identify the key elements in numerical sequences. For the availability of finding crucial factors, it is widely used in regional economics.
Let $\mathbf{C}$ denote the coefficient matrix of an economy with $n$ sectors (it can be technical coefficient matrix, or other matrixes); $C=\left(c_{i j}\right)_{n \times n}$. According to the sequence from big to small order, rearrange $c_{11}, c_{12}, \ldots, c_{n n}$ and get the vector $\mathbf{C}^{*}$. Denote the $l$ th element of $\mathbf{C}^{*}$ by $c_{i j}$; then the Weaver index is

$$
w(l)=\sum_{k=1}^{n^{2}}\left[s(k, l)-100 \times \frac{c_{i j}}{\sum_{j=1}^{n} \sum_{i=1}^{n} c_{i j}}\right]^{2},
$$

where

$$
s(k, l)= \begin{cases}\frac{100}{l} & (k \leq l) \\ 0 & (k>l) .\end{cases}
$$

Let $w\left(l^{*}\right)=\min \{w(1), w(2), \ldots, w(n \times n)\}$; the $l^{*}$ th element of $\mathbf{C}^{*}, c^{*}$, is the threshold value. If $c_{i j} \geq c^{*}$, there would be an arc from sector $i$ to sector $j$ in the industrial network with the direction in keeping with the flow. Denote the adjacency matrix by $\mathbf{W}$; then

$$
w_{i j}= \begin{cases}1, & \text { if } c_{i j} \geq c^{*} \\ 0, & \text { if } c_{i j}<c^{*}\end{cases}
$$

\section{OECD Sector Classification}

See Table 3.

\section{Competing Interests}

The authors declare that they have no competing interests.

\section{Acknowledgments}

This work was supported by the National Natural Science Foundation of China (no. 71371108).

\section{References}

[1] X. Su, P. Shi, L. Wu, and Y.-D. Song, "Fault detection filtering for nonlinear switched stochastic systems," IEEE Transactions on Automatic Control, vol. 61, no. 5, pp. 1310-1315, 2015.

[2] X. Su, L. Wu, P. Shi, and C. L. Chen, "Model approximation for fuzzy switched systems with stochastic perturbation," IEEE Transactions on Fuzzy Systems, vol. 23, no. 5, pp. 1458-1473, 2015.

[3] D. Acemoglu, V. Carvalho, A. Ozdaglar et al., "The network origins of aggregate fluctuations," Econometrica, vol. 80, no. 5, pp. 1977-2016, 2012.

[4] C. A. Hidalgo, B. Klinger, A.-L. Barabási, and R. Hausmann, "The product space conditions the development of nations," Science, vol. 317, no. 5837, pp. 482-487, 2007.

[5] B. Zhao, W. Xiao, R. Tong et al., "Blue economic connotation in industrial network perspective and its correlation structure effect-a case study of Shandong Province," China Soft Science, no. 07, pp. 135-147, 2015 (Chinese).

[6] J. McNerney, B. D. Fath, and G. Silverberg, "Network structure of inter-industry flows," Physica A: Statistical Mechanics and its Applications, vol. 392, no. 24, pp. 6427-6441, 2013. 
[7] M. Fischer-Kowalski, "On the history of industrial metabolism," in Perspectives on Industrial Ecology, D. Bourg and S. Erkman, Eds., Greenleaf Publishing, Sheffield, UK, 2003.

[8] J. Campbell, The relevance of input-output analysis and digraph concepts to growth pole theory [Ph.D. thesis], Department of Geography University of Washington, 1970.

[9] J. Campbell, "Growth pole theory, digraph analysis and interindustry relationships," Tijdschrift voor Economische en Sociale Geografie, vol. 63, no. 2, pp. 79-87, 1972.

[10] A. Morillas, L. Robles, and B. Diaz, "I-O coefficients importance: a fuzzy logic approach," International Journal of Uncertainty, Fuzziness and Knowledge-Based Systems, vol. 19, no. 6, pp. 1013-1031, 2011.

[11] B. Zhao, X. Chen, and J. Zhang, "Cycle degree of an industry and its algorithm," System Engineering-Theory \& Practice, vol. 36, no. 6, pp. 1388-1397, 2014 (Chinese).

[12] B. Zhao and J. Zhang, Introduction to Industrial Network Theory, Economic Science Press, Beijing, China, 2013 (Chinese).

[13] L. C. Freeman, "Centrality in social networks conceptual clarification," Social Networks, vol. 1, no. 3, pp. 215-239, 1978.

[14] F. Harary, Graph Theory, Addison Wesley, London, UK, 1969.

[15] Y. F. Lin, F. Cai, and Z. Li, China's Miracle: The Development Strategy and the Economic Reform, Shanghai People's Publishing House, Shanghai, China, 1994 (Chinese).

[16] H. Fehr, S. Jokisch, and L. J. Kotlikoff, "Will China eat our lunch or take us out to dinner? Simulating the transition paths of the US, EU, Japan, and China," NBER Working Paper 11668, NBER, 2005.

[17] J. Ren, W. Li, Y. Wang, and L. Zhou, "Graph-mine: a key behavior path mining algorithm in complex software executing network," International Journal of Innovative Computing, Information and Control, vol. 11, no. 2, pp. 541-553, 2015.

[18] H. He, J. Wang, and J. Ren, "Measuring the importance of functions in software execution network based on complex network," International Journal of Innovative Computing, Information and Control, vol. 11, no. 2, pp. 719-731, 2015.

[19] X. Chen and B. Zhao, "Research on industry linkage correction influence coefficients based on hypothetical extraction method," Management Review, vol. 26, no. 6, pp. 23-32, 2014 (Chinese).

[20] B. Zhao, C. Yin, and J. Zhang, "A study on industry complex network and its modeling - on the example of Shandong province," Economic Management, no. 7, pp. 139-148, 2011 (Chinese).

[21] S. Schultz, "Approaches to identifying key sectors empirically by means of input-output analysis," The Journal of Development Studies, vol. 14, no. 1, pp. 77-96, 2007.

[22] G. Cella, "The input-output measurement of interindustry linkages," Oxford Bulletin of Economics and Statistics, vol. 46, no. 1, pp. 73-84, 1984.

[23] B. J. Clements, "On the decomposition and normalization of interindustry linkages," Economics Letters, vol. 33, no. 4, pp. 337340, 1990.

[24] E. Dietzenbacher and J. A. Van Der Linden, "Sectoral and spatial linkages in the EC production structure," Journal of Regional Science, vol. 37, no. 2, pp. 235-257, 1997.

[25] R. E. Miller and M. L. Lahr, "A taxonomy of extractions," in Regional Science Perspectives in Economic Analysis, Elsevier, Amsterdam, The Netherlands, 2001.

[26] J. Cai and P. Leung, "Linkage measures: a revisit and a suggested alternative," Economic Systems Research, vol. 16, no. 1, pp. 65-86, 2004. 

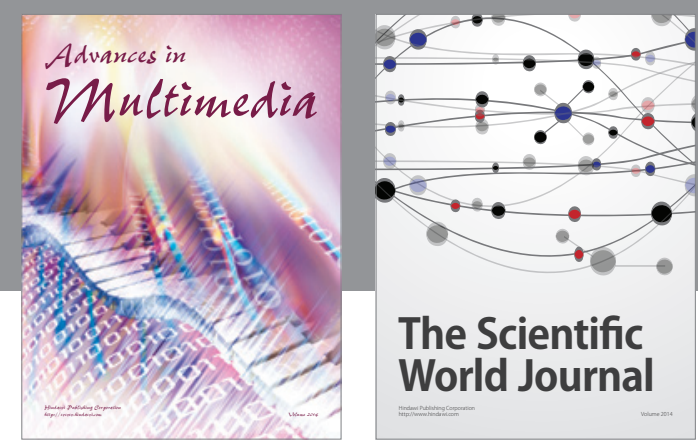

The Scientific World Journal
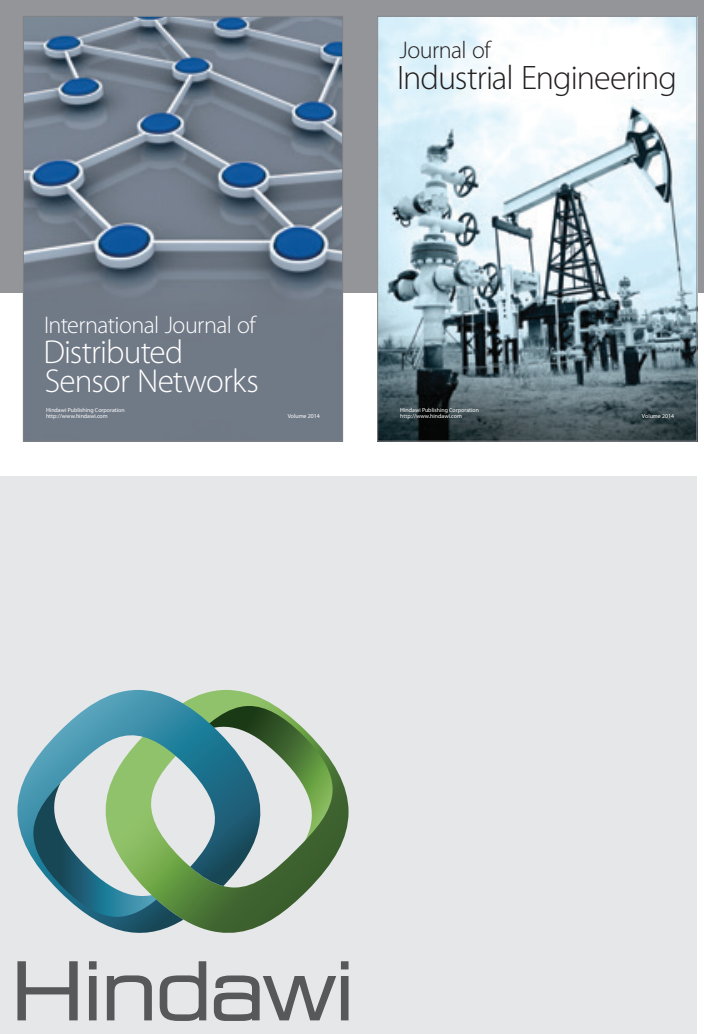

Submit your manuscripts at

http://www.hindawi.com

\section{Computer Networks} and Communications
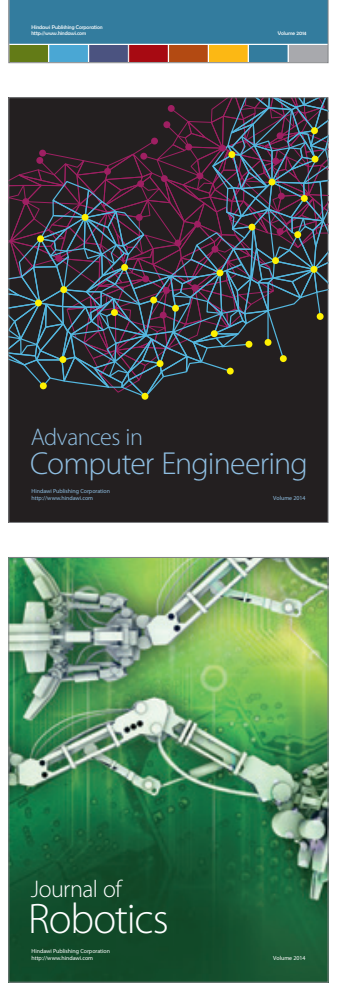
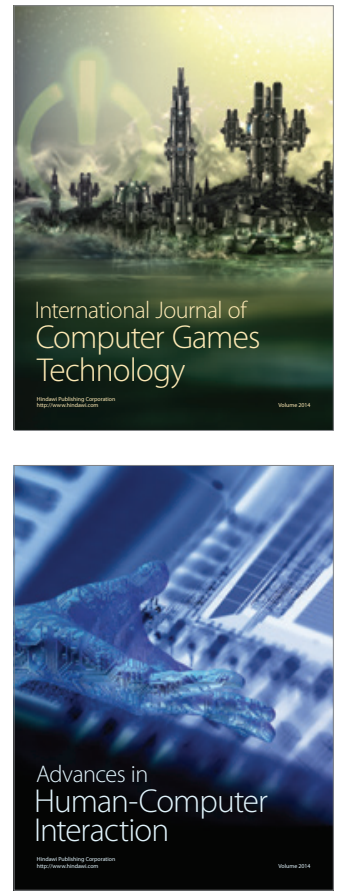
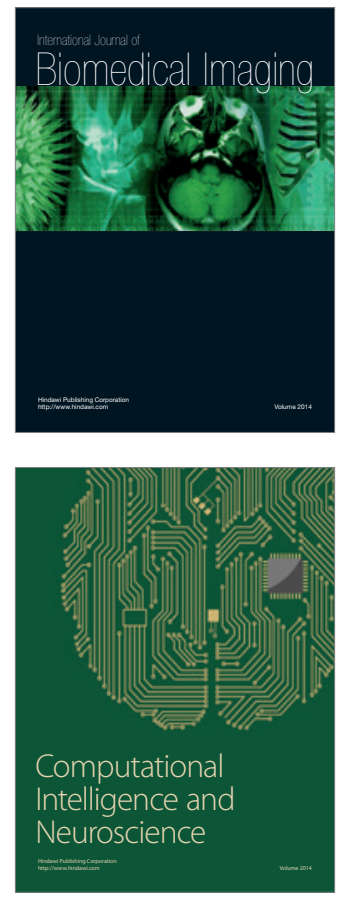
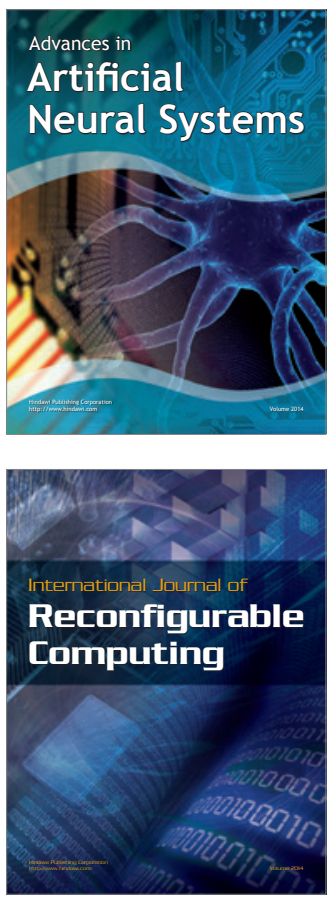
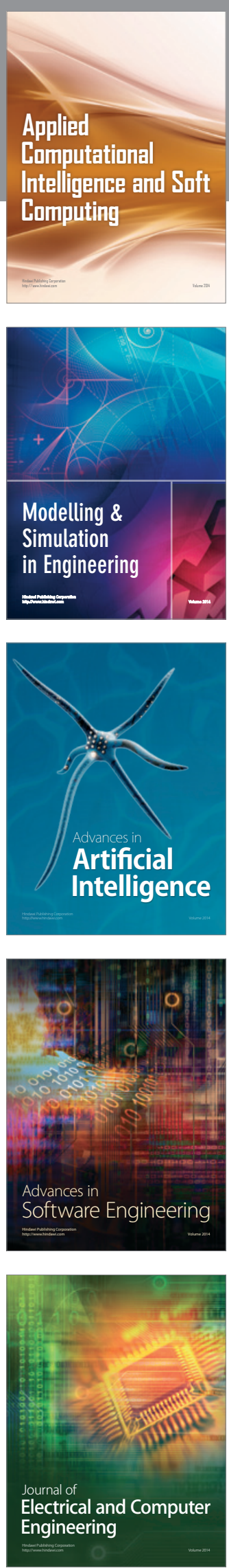